\title{
Seated Transforaminal Injection Approach in Severe Lumbar Stenosis
}

\author{
Jason S. Lipetz, MD*, Sarjoo Bhagia, MD**, and Thomas J. Dicarlo, LRT"
}

The use of seated positioning for the performance of a fluotion is presented. An 81 year old gentleman presented with a lumbar radiculopathy with radiographs demonstrating foraminal stenosis arising in the setting of offending disc pathology, multilevel and advanced central canal compromise, and a scoliotic deformity. He was unable to tolerate prone positioning secondary to radicular pain. A seated approach allowed for the patient to remain comfortable, and a satisroscopically guided transforaminal lumbar epidural injec-

factory epidurogram was observed. Comparative images of the L4 transforaminal injection performed in the prone and seated position may be consistent with an increased foraminal cross sectional area in the seated and forward flexed posture.

Keywords: Transforaminal injection, epidural, lumbar radiculopathy, fluoroscopy, lumbar spinal stenosis

Epidural injections for the treatment of lumbar and radicular pain have been described in the literature since 1901 (1). Injection of corticosteroids into the epidural space as a treatment of lumbosacral radicular pain was initially described in 1952 (2). Epidural injection can be achieved through a caudal, translaminar, or transforaminal approach (3). Translaminar injections without fluoroscopic guidance are typically performed with the patient in the lateral decubitus or sitting position (4). Fluoroscopically guided transforaminal lumbar injections are commonly performed with the patient prone (5). We present a seated fluoroscopically guided transforaminal lumbar injection approach, which has been employed in a severely stenotic patient who proved unable to tolerate prone positioning.

\section{CASE REPORT}

We report the case of an 81-year old gentleman who initially presented with the chief complaint of right lower limb pain. His symptoms began 12 days prior to his visit while performing repetitive bending and lifting maneuvers dur-

From the Department of Rehabilitation Medicine, North Shore - Long Island Jewish Health System, East Meadow, NY and Department of Physical Medicine and Rehabilitation, Temple University Hospital, Philadelphia, PA. *Director of Center for Spine Rehabilitation, North Shore. **Chief resident and clinical instructor of the Temple University Hospital. "Radiological coordinator, North Shore. Address correspondence: Jason S. Lipetz, MD, Center for Spine Rehabilitation, NS-L1J Health System, 801 Merrick Avenue, East Meadow, NY 11554. E-mail: JLipetz@lij.edu ing gardening. His treatment prior to this visit included an oral steroid taper, increasing dosages of oral analgesics, a home TENS unit, and continuing physical therapy efforts. His chief area of discomfort was noted to affect the anterior thigh, generally terminating at the knee. He described parasthesias and numbness affecting the anteromedial calf. His visual analogue score ranged from 8 to 10 . His symptoms were exacerbated by prolonged standing and some relief was realized with forward flexed postures. He had been utilizing a walker over the past week as well as a patellofemoral brace with lateral hinges. He described a complete discontinuation of his previous regular swimming and resistance training regimen. His sleep was regularly interrupted by lower limb pain.

His medications included Prevacid $\AA$, Proventil $\AA$, Flomax ${ }^{\circledR}$, OxyContin ${ }^{\circledR}$, and methylprednisolone. His past medical history was significant for benign prostatic hypertrophy, asthma, peptic ulcer disease and an arrhythmia. He had received a permanent pacemaker, and multiple prior skin biopsies revealed benign lesions. He specifically denied bowel or bladder incontinence and reported no constitutional symptoms. He described no history of endocrine illness. His respiratory status had been stable. The patient was a non-smoker, married with two children, and continued to work part time as a physician.

On physical examination, the patient's height was 6'0", weight $180 \mathrm{lbs}$., pulse 72, respirations 18. Anterior thigh atrophy was noted on the right where muscle girth measured $18 \mathrm{~cm}$ proximal to the mid-patella was $42 \mathrm{~cm}$ on the right and $44.5 \mathrm{~cm}$ on the left. Distal pulses were intact. Passive range of motion of the right hip was not pain pro- 
voking. Pelvic rocking and sustained hip flexion maneuvers resulted in lumbar pain. A reverse straight leg raise maneuver was avoided, and a straight leg raise maneuver resulted in terminal range buttock pain. Toe walking was performed with difficulty on the left, and heel and tandem walking were performed with a balance deficit. Sharp sensation was diminished over the right anteromedial calf. A left patella reflex was graded $2 / 4$ and the right was absent. The left Achilles reflex was absent and the right trace. Plantar responses were downward going and no clonus was observed. Right knee extension strength was graded $4 / 5$, ankle dorsiflexion 4+ to 5-, hip flexion 3+, extensor hallucis $4+$, and hip abduction 4+. Active lumbar extension and side bending were limited to approximately 10 degrees secondary to thigh pain.

Plain radiographs of the lumbar spine demonstrated a marked lumbar kyphoscoliotic deformity with an apex at L3, partial lumbarization of the S1 body, and aortic sclerosis. A multiplanar reformatted lumbar $\mathrm{CT}$ revealed a grade II/III spondylolisthesis at L5-S1, a grade I spondylolisthesis at L4-5, a vacuum disc at L3-4, moderate to severe central stenosis at L3-4 arising in the setting of protruding disc material and advanced posterior element arthrosis, a right posterolateral and foraminal disc protrusion at this level noted to markedly compromise the neural foramen, severe central stenosis at L4-5, with similar foraminal disc pathology to the right at this level, and moderate central stenosis and thecal sac compromise at L5-S1 arising from a central protrusion.

Our impression following his initial evaluation was that the patient was experiencing symptoms arising from a lumbar radiculopathy of L4 and potentially L3 origin. While his predominantly proximal lower limb weakness may have been consistent with an L3 radiculopathy, his anteromedial calf complaints were more consistent with L4 involvement. Combined root pathology could be explained by his foraminal disc pathology to the right at both L3-4 and L4-5. Alternatively, the lateral disc lesion and advanced central stenosis at L3-4 could similarly affect both the L3 and L4 roots. The patient did not wish to proceed with electrodiagnostic testing. Additionally, the relative acuity of his presentation may have resulted in a less diagnostic needle exam of the lower limb. Rather than employ diagnostic selective spinal nerve injections, it was decided to proceed with therapeutic transforaminal injections at the L3 and L4 levels. A flexion based spine stabilization program was initiated and strengthening of the hip flexors and knee extensors was to be graduated as his symptoms allowed. It was our plan to taper his oxycodone following

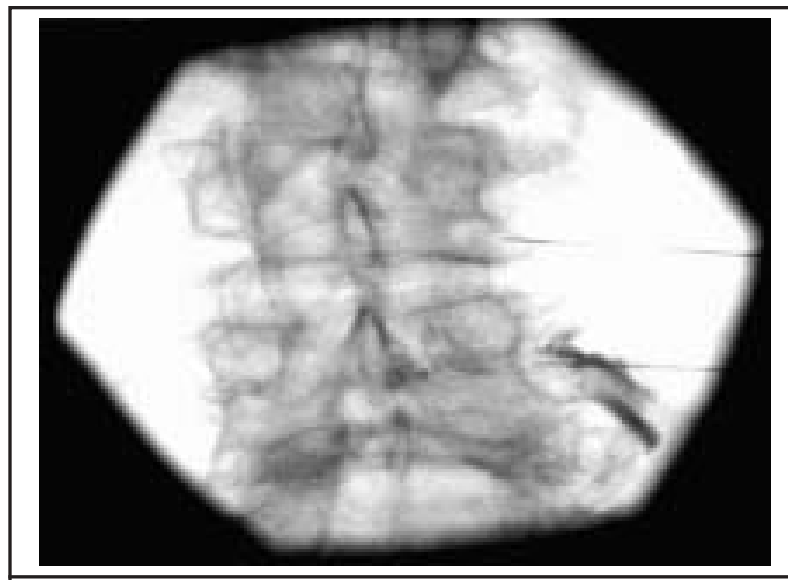

Fig. 1. Prone position right L4 epidurogram and spinal needle approaching right L3 foramen.

the introduction of injections. We wished to avoid surgery if at all possible, as his case would require a more extensive multilevel decompression and likely stabilization procedure.

His first injection was performed four days later. Positioning proved difficult, as the patient experienced thigh pain when attempting to lie prone. We were able to achieve adequate oblique and postero-anterior (PA) imaging with the patient in a partial lateral decubitus position. To minimize procedural time, both the L3 and L4 needles were placed during oblique imaging and then further advanced with PA visualization. The resultant epidurogram during L4 injection is demonstrated in Fig. 1. Some cephalad spread along the medial pedicular border was noted with less contrast appreciated foraminally beneath the pedicle.

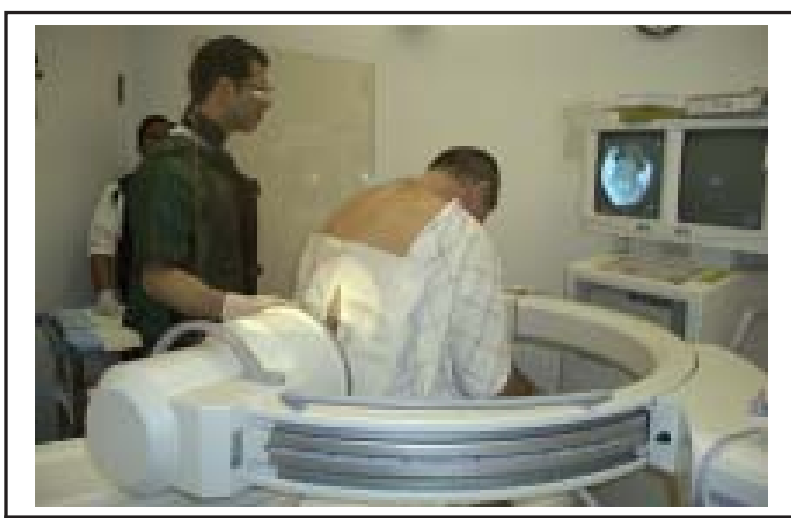

Fig. 2. Patient demonstrated in seated and forward flexed position for performance of fluoroscopically guided transforaminal lumbar injection. 


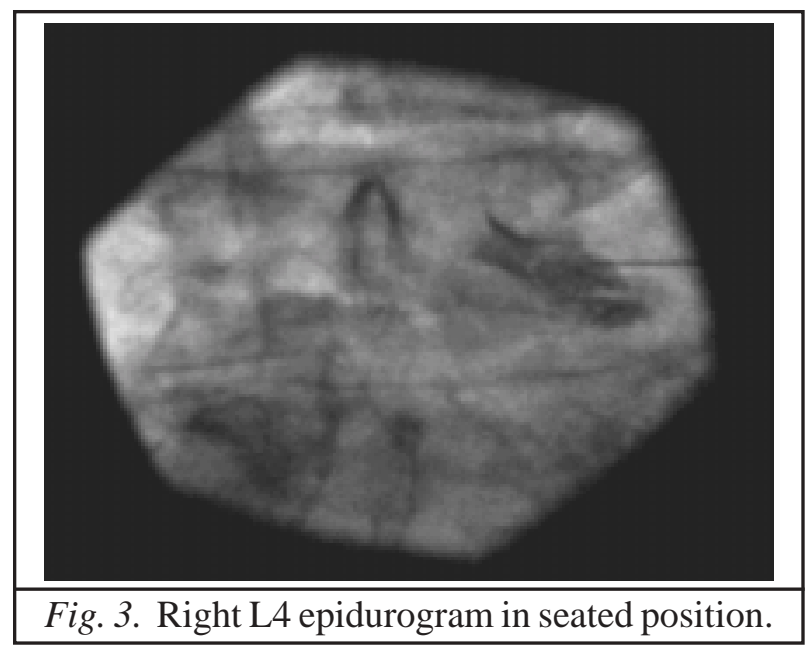

Most contrast was observed to spread in the more lateral foramen and extraforaminally. With further needle advancement, the patient began to experience limb discomfort. This dye pattern, particularly in the setting of a suspected offending foraminal disc, was considered satisfactory. Interestingly, during the L3 injection, his anterior thigh complaints were transiently reproduced in a concordant fashion with the introduction of injectate. This was the first of two therapeutic injections at L3 and L4, which resulted in one year of symptomatic relief.

After this period of time, following a lifting maneuver, the patient experienced a symptomatic recurrence, which was less pronounced than his initial episode. An additional injection was scheduled. This time, the patient was unable to achieve prone positioning and tended to curl in a fetal position to avoid inciting his radicular thigh complaints. We attempted to reverse the position of his head and feet as well as modified decubitus postioning on the left and right. In the setting of the C-arm's inability to revolve beyond 120 degrees and the radio-opaque table bars, adequate visualization could not be achieved. At this point we had the patient assume a seated and forward flexed posture at the table end, which proved quite comfortable. The axis of rotation of the $\mathrm{C}$-arm was modified as depicted in Fig. 2. Adequate visualization was achieved. The resultant epidurogram at the L4 level is demonstrated in Fig. 3 , with noticeably more contrast remaining within the midportion of the foramen. This was the first of two injections, which has similarly resulted in an additional six months of satisfactory relief.

\section{DISCUSSION}

Transforaminal injections at the level of the first sacral nerve root were initially descried in the European literature in the 1950's $(2,6)$. In 1971, Macnab (7) reported the value of selective nerve root infiltration in the diagnosis of radiculopathy, and the technique of transforaminal injections was further described by Tajima et al (8) in 1980 and Derby et al (9) in 1993.

Lumbosacral transforaminal injections offer distinct technical advantages over the traditional translaminar or caudal approach. Transforaminal injections are performed with fluoroscopic guidance. The miss rate in non-fluoroscopically guided translaminar and caudal injections performed by experienced clinicians has been estimated at $25-38 \%$ $(10,11)$. Fluoroscopy also allows the physician to detect inadvertant intravascular injection which can occur in $8 \%$ of lumbar and $21 \%$ of sacral injections without reliable detection by aspiration or syringe inspection (12). The transforaminal approach allows for greater control of medication dispersal and the introduction of injectate immediately surrounding the affected nerve root (13)

In a study of 28 patients with severe lumbar radiculopathy arising in the setting of foraminal disc pathology, 22 were successfully treated with transforaminal injections with relief documented for an average of 3.4 years (14). In a prospective, randomized, double blind, controlled study by Riew et al (15), a statistically significant response to transforaminal injections was observed with $71 \%$ of patients in the treatment group avoiding surgical decompression.

The patient in this case presented to our office specifically to review his candidacy for transforaminal injections as a final nonsurgical treatment option. His first series of injections provided relief lasting for one year. Upon the recurrence of less pronounced radicular pain, he was unable to tolerate the prone positioning typically employed for the transforaminal injection approach. With a pronounced scoliosis, multilevel listhesis, and advanced central canal and neural foraminal compromise, the patient was only able to tolerate seated positioning for subsequent injection procedures. His more acute foraminal disc pathology likely contributed as a mechanical stressor, which prohibited him for introducing the relative lumbar extension associated with prone positioning.

The intervertebral foramen is shaped similar to an inverted teardrop with its height and cross sectional area ranging from 11 to $19 \mathrm{~mm}$ and 40-160mm2 (16). Dynamic imaging studies have demonstrated a $12-19 \%$ increase in foraminal cross- sectional area with flexion and a $15-23 \%$ 
reduction with extension $(17,18)$. Studies by Panjabi et al (19) and Nowicki et al (20) suggest that degenerative spinal segments with less inherent stiffness demonstrate enhanced kinematics and more pronounced dynamic foraminal stenosis with extension, lateral bending and rotational maneuvers. More recent biomechanical studies have confirmed reduced foraminal dimensions not only with extension, but also with ipsilateral axial rotation and sidebending (21). In our patient with scoliosis and stenosis, seated postioning may have resulted in increased foraminal dimensions through a relief of extension and rotational stressors. While a definitive statement can not be made regarding any change in our patient's right L4 neural foraminal dimensions with seated positioning, his comfort level and enhanced foraminal filling during contrast administration may speak toward an increased cross- sectional area. We describe this seated transforaminal approach as a means of maximizing patient comfort and perhaps performing a technically superior injection in individuals with severe stenosis and radicular pain which prohibits them from maintaining more traditional procedural positioning.

\section{REFERENCES}

1. Cathelin F. Mode d'action de la cocaine injecte dons l'escape epidural par le proceda dee canal sacre. Curr Opin Soc Biol 1901; 53:478-479

2. Robechhi A, Capre R. L'idrocortisone (composto F). Prime esperienze cliniche in campo reumatologico. Minerva Med 1952; 98:1259-63.

3. Weinstein SM, Herring SA, Derby R. Contemporary concepts in spine care: Epidural steroid injections. Spine 1995; 20:1842-1846.

4. Brown DL. Spinal, epidural and caudal anesthesia. In Miller RD (ed.), Anesthesia $5^{\text {th }}$ ed. Churchill Livingstone, Edinburgh, 2000, pp 1491-1519.

5. Manchikanti L. Transforaminal lumbar epidural steroid injections. Pain Physician 2000; 3:374-398.

6. Lievre JA, Block-Mechel H, Pean G, et al. L'hydrocortisone en injection locale. Rev Rheum 1953; 20:310-311.

7. Macnab I. Negative disc exploration: An analysis of the causes of nerve-root involvement in sixty-eight patients. J Bone Joint Surg 1971; 53:891-903.

8. Tajima T, Furukawa K, Kuramochi E. Selective lumbosacral radiculography and block. Spine 1980; 5:6877 .

9. Derby R, Bogduk N, Kine G. Precision percutaneous blocking procedures for localizing spinal pain. Part 2:
The lumbar neuraxial compartment. Pain Digest 1993; 3:175-188

10. Renfrew DL, Moore TE, Kathol MH et al. Correct placement of epidural steroid injections: fluoroscopic guidance and contrast administration. Am J Neuroradiol 1991; 12:1003-1007.

11. White AH. Injection techniques for the diagnosis and treatment of low back pain. Orthop Clin North Am $1983 ; 14: 553-567$.

12. Furman MB. O'Brien EM. Zgleszewski TM. Incidence of intravascular penetration in transforaminal lumbosacral epidural steroid injections. Spine 2000; 25:2628-2632.

13. Bogduk N, Christophidis N, Cherry D et al. Epidural steroids in the management of back pain and sciatica of spinal origin. Report of working party on epidural use of steroids in the management of back pain. National Health and Medical Research Council, Canberra, Australia 1993.

14. Weiner BK, Fraser RD. Foraminal injection for lateral lumbar disc herniation. J Bone Joint Surg 1997; 79-B:804-807.

15. Riew KD, Yin Y, Gilula L et al. The effect of nerveroot injections on the need for operative treatment of lumbar radicular pain. A prospective, randomized, controlled, double-blind study. J Bone Joint Surg Am 2000; 82-A:1589-1593.

16. Stephens MM, Evans JH, O’Brien JP. Lumbar intervertebral foramens: An in vitro study of their shape in relation to intervertebral disc pathology. Spine 1991; 16: 525-529.

17. Inufusa A, An HS, Lim TH et al. Anatomic changes of the spinal canal and intervertebral foramen associated with flexion-extension movement. Spine 1996; 21:2412-2420.

18. Schmid MR, Stucki G, Duewell S et al. Changes in cross-sectional measurements of the spinal canal and intervertebral foramen as a function of body position: In vivo studies on an open-configuration MR system. AJR Am J Roentgenol 1999; 172: 1095-1102.

19. Panjabi MM, Takata K, Goel VK. Kinematics of lumbar intervertebral foramen. Spine 1983; 8:348-357.

20. Nowicki BH, Haughton VM, Schmidt TA et al. Occult lumbar lateral spinal stenosis in neural foramina subjected to physiologic loading. AJNR Am J Neuroradiol 1996; 17:1605-1614.

21. Fujiwara A, An HS, Lim T et al. Morphologic changes in the lumbar intervertebral foramen due to flexionextension, lateral bending, and axial rotation: An In Vitro Anatomic and Biomechanical Study. Spine 2001; 26: $876-882$. 